\title{
Ajuste do modelo Chistiansen-Hargreaves para estimativa da evapotranspiração do feijão no cerrado ${ }^{1}$
}

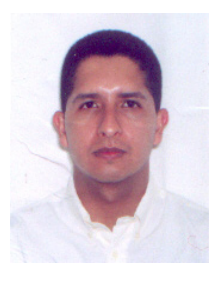

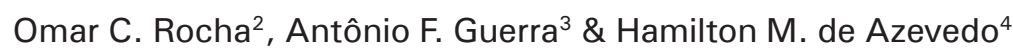

${ }^{1}$ Convênio UFPB/Embrapa Cerrados, parte da dissertação de Mestrado apresentada pelo primeiro autor

${ }^{2}$ M.Sc. Rua Siqueira Campos 1353, Recreio, CEP 45020.505, Vitória da Conquista, BA. Fone: (77) 9136-4910.

E-mail: omarcr2@ig.com.br (Foto)

${ }^{3}$ M.Sc., PhD. Pesquisador da Embrapa Cerrados, CP 8233, CEP 73301-970, Planaltina, DF. Fone: (61) $388-9862$.

E-mail: guerra@cpac.embrapa.br

${ }^{4}$ UFCG/CCT/DEAg. CP 10087, CEP 58109-970, Campina Grande, PB. Fone: (83) 310-1318. E-mail: hamilton@deag.ufcg.edu.br

Protocolo $49-11 / 5 / 2000$ - Aprovado em 15/8/2003

\begin{abstract}
Resumo: Os produtores de feijão da região do Cerrado contam com apenas uma tecnologia, já estabelecida, para o manejo das irrigações: a tensiometria. Muito embora essa metodologia tenha alto potencial de uso não tem sido amplamente adotada pelos produtores, razão pela qual a utilização de modelos de estimativa de evapotranspiração tem se mostrado bastante aplicável à realidade da região. Assim, este trabalho tem o objetivo de avaliar o desempenho do modelo Chistiansen-Hargreaves na estimativa da evapotranspiração da cultura do Feijão Preto, no período seco do Cerrado brasileiro, almejando colocar à disposição dos produtores um modelo ajustado, que permita um manejo eficiente da irrigação no sistema produtivo da região. A evapotranspiração do feijoeiro foi medida com um lisímetro de pesagem. O experimento foi conduzido na Embrapa Cerrados, localizada em Planaltina, DF, Brasil. Quando calculado com coeficientes de cultura determinados na pesquisa e testado com o termo energético ajustado $\left(S_{0}=0,5\right)$ o modelo apresentou ótimo desempenho podendo, nesta condição, ser empregado com segurança no manejo de irrigação.
\end{abstract}

Palavras-chave: evapotranspiração, lisímetro de pesagem, manejo de irrigação, Phaseolus vulgaris

\section{Chistiansen-Hargreaves model adjustment for estimating evapotranspiration of bean crop in the Cerrado region}

\begin{abstract}
Bean producers from the Brazilian Cerrado region have only one technology for the irrigation management: the measurement of the water tension in the soil through the use of tensiometers. Although this methodology has high potential, it has not been widely adopted by the producers. Thus, the utilization of models to estimate evapotranspiration estimate has shown to be applicable to the Cerrado region. So, this paper aims to evaluate the performance of the Chistiansen-Hargreaves model to estimate evapotranspiration of black bean crop in the dry season of the Brazilian Cerrado region. It also aims to provide producers an the adjusted model to estimate evapotranspiration which permit an efficient management for the agricultural irrigated system of the Cerrado region. The evapotranspiration of the black bean crop was measured with the use of a weighing lysimeter. The experiment was carried in the Brazilian Agricultural Research Corporation (Embrapa Cerrados), located in Planaltina, DF, Brazil. When calculated with crop coefficient, determined in the research and tested with adjusted energetic term $\left(S_{0}=0.5\right)$, the ChistiansenHargreaves model presented an excellent performance and may be used in irrigation management.
\end{abstract}

Key words:, evapotranspiration, weighting lysimeter, irrigation handling, Phaseolus vulgaris

\section{INTRODUÇÃO}

A região de cerrado da América do Sul representa, potencialmente, uma das mais aptas e importantes para a exploração agrícola. A distribuição geográfica dos seus 250 milhões de hectares abrange a Venezuela, Colômbia, Bolívia e Brasil, com 204 milhões de hectares (Macedo, 1997).
As características edafoclimáticas da região permitem o desenvolvimento, durante a estação chuvosa, da maior parte das culturas, mas, persistem problemas devido à existência de período seco (maio a setembro) e da irregularidade das chuvas no período de máximo desenvolvimento das culturas ("veranico") resultando em necessidade de irrigação total no período seco, e suplementar no período chuvoso. 
Uma das grandes dificuldades dos produtores do Cerrado encontra-se no manejo da irrigação, já que, na maioria dos casos, a água é aplicada sem nenhum critério de monitoramento, resultando no uso inadequado dos recursos hídricos. Vários são os procedimentos que podem ser utilizados como critério para o manejo de água da irrigação, em que teoricamente, o melhor critério seria aquele que considera o maior número de fatores que determinam a transferência de água no sistema solo-planta-atmosfera.

Os critérios de manejo de água normalmente utilizados são, via de regra, baseados em medidas no solo, na planta e na atmosfera. Os baseados em medidas no solo se fundamentam na determinação direta ou indireta do teor de água presente no substrato, enquanto os baseados em medidas na planta se estabelecem no monitoramento do potencial hídrico, da resistência estomática, da temperatura da folha por meio de termômetro infravermelho e outros; já os baseados em medidas climáticas variam desde simples medidas de evaporação de água de um tanque, como o Classe $\mathrm{A}$, até complexas equações para estimativa da evapotranspiração. A grande limitação dessas equações encontra-se na precisão das estimativas, que dependem diretamente da acurácia das variáveis e da precisão desses modelos, os quais vêm sendo utilizados em condições climáticas e agronômicas muito diversas daquelas em que foram concebidas.

Dentre os diversos métodos de determinação da evapotranspiração um dos mais precisos é o da utilização de lisímetros de pesagem, todavia, essa tecnologia restringe-se a instituições de pesquisa, devido à sua pouca aplicabilidade econômica em áreas agrícolas; desta forma, a estimativa, a partir de modelos matemáticos, tem recebido bastante atenção pelo meio agrícola, em virtude da praticidade e da facilidade oferecidas por alguns modelos; entretanto, existe necessidade de se ajustar esses modelos para as diversas regiões produtoras, já que, em suas formas originais, pouquíssimas serão as estimativas compatíveis com as condições climáticas locais.

Nos últimos anos um grande número de trabalhos têm sido direcionados para o estudo da estimativa da evapotranspiração por meio de modelos; todavia, numa avaliação das equações mais comuns para a determinação da evapotranspiração, feita pela American Association of Civil Engineers, na qual se utilizaram dados climáticos de 11 diferentes localidades do mundo, concluiu-se que não existe um método, que utilize dados climáticos, que possa ser globalmente adequado, sem que exista, também, algum tipo de calibração local ou regional, principalmente em regiões tropicais e regiões altas (Jensen et al., 1989). Informação que é validada devido ao fato de que, em condições tropicais, existe uma grande demanda de energia do sistema para o fluxo de calor latente, o que ocorre em menor intensidade em climas úmidos.

Atualmente, os produtores irrigantes da região do Cerrado contam com apenas uma tecnologia, já estabelecida, para o manejo das irrigações, que é a medição da tensão de água no solo por meio de tensiômetros (Guerra \& Silva, 1998). Essa metodologia, apesar de ter demonstrado alto potencial de uso, não tem sido amplamente adotada pelos produtores, por necessitar de um número expressivo de instrumentos para representar cada área irrigada, cujo problema é agravado pela necessidade constante de cuidados com os tensiômetros, o que muitas vezes, os produtores não têm condição de satisfazer, razão pela qual a utilização de modelos de estimativa de evapotranspiração tem-se mostrado bastante aplicável à realidade da região; entretanto, o uso desses modelos sem uma prévia avaliação, pode resultar na obtenção de estimativas imprecisas, o que poderia desacreditar a tecnologia junto aos produtores.

No Brasil, a cultura do feijão (Phaseolus vulgaris L.) tem sido objeto de diversos estudos por se tratar da principal fonte da dieta alimentar nacional com um consumo médio "per capita" de $16 \mathrm{~kg} \mathrm{ano}^{-1}$ (Roston, 1990), ocupando uma área de, aproximadamente, 3,5 milhões de hectares (BRASIL. Ministério da agricultura, 1996).

A maior parte da produção de feijão irrigado no Cerrado do Brasil central ocorre de maio a setembro, período caracterizado pela ausência de chuvas e por condições reduzidas de umidade relativa, motivo pelo qual este trabalho objetiva avaliar o desempenho do modelo Chistiansen-Hargreaves na estimativa da evapotranspiração da cultura do Feijão Preto, no período seco do Cerrado brasileiro, almejando colocar à disposição dos produtores, um modelo ajustado, que permita um manejo eficiente da irrigação no sistema produtivo da região.

\section{MATERIAL E MÉTODOS}

\section{Localização do experimento e características da área}

As medidas dos parâmetros vegetativos e climáticos foram obtidas na Empresa Brasileira de Pesquisa Agropecuária Centro de Pesquisa Agropecuária dos Cerrados, Planaltina, DF - sobre Feijão Preto (Phaseolus vulgaris L.), cultivado em um Latossolo Vermelho Escuro Argiloso, no período de 23 de junho a 05 de setembro de 1999 (período seco).

$\mathrm{O}$ ensaio foi instalado em uma área com aproximadamente 09 ha, dotada de um lisímetro de pesagem e irrigada por um equipamento de irrigação por aspersão, do tipo pivô central.

Planaltina encontra-se localizada a uma latitude de $15^{\circ} 35^{\prime} 30^{\prime \prime}$ $\mathrm{S}$, longitude de $47^{\circ} 42^{\prime} 30^{\prime \prime} \mathrm{W}$ e altitude de $1007 \mathrm{~m}$, apresentando clima, segundo a classificação de Köppen, do tipo $\mathrm{CWh}_{1}$, com precipitação média anual de $1.460 \mathrm{~mm}$.

\section{Condução da cultura}

Plantio: Utilizou-se a variedade de feijão "Diamante Negro", indicada para lançamento na V Reunião da comissão técnica da região II, em Goiânia - GO na Empresa Brasileira de Pesquisa Agropecuária/Centro Nacional de Pesquisa do arroz e feijão (CNPAF) em junho de 1991.

A semeadura foi realizada no dia 04 de junho de 1999, manualmente no interior do lisímetro e mecanicamente no restante da área, sendo distribuídas aproximadamente, 12 sementes viáveis por metro linear de sulco, no espaçamento de $45 \mathrm{~cm}$ entre linhas.

Tratos culturais: Com base na análise química do solo, aplicaram-se, como adubação de plantio, $400 \mathrm{~kg} \mathrm{ha}^{-1}$ da fórmula 4-30-16 + Zn e, via água de irrigação, $70 \mathrm{~kg} \mathrm{ha}^{-1} \mathrm{de} \mathrm{N}$ na forma de uréia, distribuídos aos 26 e 42 dias após plantio. 
Visando-se controlar ervas daninhas, aplicou-se, 24 dias após o plantio, 1,8 $\mathrm{L} \mathrm{ha}^{-1}$ de Fusiflex, mecanicamente em toda a área do pivô, à exceção do interior do lisímetro onde a limpeza foi manual.

No início do estádio reprodutivo, aos 40 dias após plantio, foi aplicado, mecanicamente, $1,8 \mathrm{~L} \mathrm{ha}^{-1}$ de Tamaron para controle da mosca branca.

Manejo da irrigação: As irrigações iniciais foram feitas aproximadamente a cada três dias com lâminas em torno de 10 a $12 \mathrm{~mm}$ com o propósito de garantir a germinação das sementes e preencher o reservatório do solo. As irrigações seguintes foram realizadas com base nas leituras diárias de três baterias de tensiômetros instalados nas profundidades de 10,20 e 30 $\mathrm{cm}$ na linha de plantio. $\mathrm{O}$ momento de irrigar foi determinado quando a leitura média dos tensiômetros instalados a $10 \mathrm{~cm}$ atingia - $40 \mathrm{kPa}$, conforme Figuerêdo et al. (1997).

A quantidade de água a ser aplicada por irrigação foi calculada para elevar a umidade do solo, na camada de 0 a 30 $\mathrm{cm}$, até a condição de capacidade de campo, o que era possível através das curvas características de umidade do solo e das leituras dos tensiômetros. Em todo ciclo do feijoeiro foram aplicados $460 \mathrm{~mm}$ de água distribuídos conforme a Tabela 1.

Tabela 1. Etapas do desenvolvimento do Feijão preto e quantidade de água aplicada durante o ciclo em Planaltina, DF

\begin{tabular}{|c|c|c|}
\hline Data & $\begin{array}{c}\text { Etapas do } \\
\text { Desenvolvimento }\end{array}$ & $\begin{array}{c}\text { Lâmina } \\
\text { Aplicada (mm) }\end{array}$ \\
\hline $04 / 06 / 99$ & \multirow[t]{3}{*}{ Plantio } & 18,00 \\
\hline 07/06/99 & & 6,30 \\
\hline 08/06/99 & & 7,88 \\
\hline $10 / 06 / 99$ & \multirow[t]{12}{*}{ Germinação } & 6,30 \\
\hline $12 / 06 / 99$ & & 6,30 \\
\hline $15 / 06 / 99$ & & 6,30 \\
\hline $18 / 06 / 99$ & & 7,88 \\
\hline 23/06/99 & & 25,20 \\
\hline $27 / 06 / 99$ & & 6,30 \\
\hline $30 / 06 / 99$ & & 6,30 \\
\hline 01/07/99 & & 25,20 \\
\hline 07/07/99 & & 25,20 \\
\hline $11 / 07 / 99$ & & 21,00 \\
\hline $16 / 07 / 99$ & & 6,30 \\
\hline $17 / 07 / 99$ & & 25,20 \\
\hline 21/07/99 & \multirow[t]{3}{*}{ Início da floração } & 21,00 \\
\hline $25 / 07 / 99$ & & 25,20 \\
\hline $30 / 07 / 99$ & & 31,50 \\
\hline 04/08/99 & \multirow[t]{4}{*}{ Início da frutificação } & 25,20 \\
\hline 08/08/99 & & 25,20 \\
\hline $12 / 08 / 99$ & & 25,20 \\
\hline $17 / 08 / 99$ & & 25,20 \\
\hline $22 / 08 / 99$ & \multirow[t]{3}{*}{ Início da maturação fisiológica } & 25,20 \\
\hline $27 / 08 / 99$ & & 31,50 \\
\hline $31 / 08 / 99$ & & 25,20 \\
\hline 05/09/99 & Colheita & - \\
\hline & Tot: & 460,06 \\
\hline
\end{tabular}

Colheita: Devido à variedade diamante negro apresentar deiscência acentuada no final do ciclo, realizou-se a colheita quando as vagens do terço médio, da maioria das plantas, atingiam a maturação fisiológica, momento em que ainda ocorria uma expressiva fotossíntese na planta.
O feijão foi colhido manualmente no interior do lisímetro e em 10 parcelas aleatórias no dia 4 de setembro e, no restante da área foi arrancado manualmente, no dia 5 de setembro de 1999 e trilhado mecanicamente quinze dias depois.

Todo o manejo da cultura foi realizado de forma a obter o potencial produtivo da variedade, o que resultou numa produtividade de, aproximadamente, 50 sacos por hectare.

\section{Instrumentação e coleta de dados}

Evapotranspiração: A evapotranspiração do feijão foi medida, diariamente, durante todo o ciclo por meio de um lisímetro de pesagem de $2 \mathrm{~m}$ de largura por $3 \mathrm{~m}$ de comprimento e 1,2 $\mathrm{m}$ de profundidade. O sistema é constituído de uma caixa metálica montada sobre quatro células de carga, conectadas a um indicador de balança EZ 210, e de um outro sistema com duas células de carga, ligadas a um outro indicador, para medir o excesso. A precisão das medidas foi programada nos indicadores de balança para $200 \mathrm{~g}$, o que corresponde, em um lisímetro de $6 \mathrm{~m}^{2}$, a uma precisão nas medidas de evapotranspiração, de $0,03 \mathrm{~mm}$.

A curva de evapotranspiração real (Et $\left.{ }_{r}\right)$ do feijão em relação aos dias após plantio (DAP) (Figura 1) foi determinada com base nas pesagens do lisímetro registradas às 8:30 h de cada dia, dividindo-se a variação ocorrida no intervalo das irrigações pela área do lisímetro.

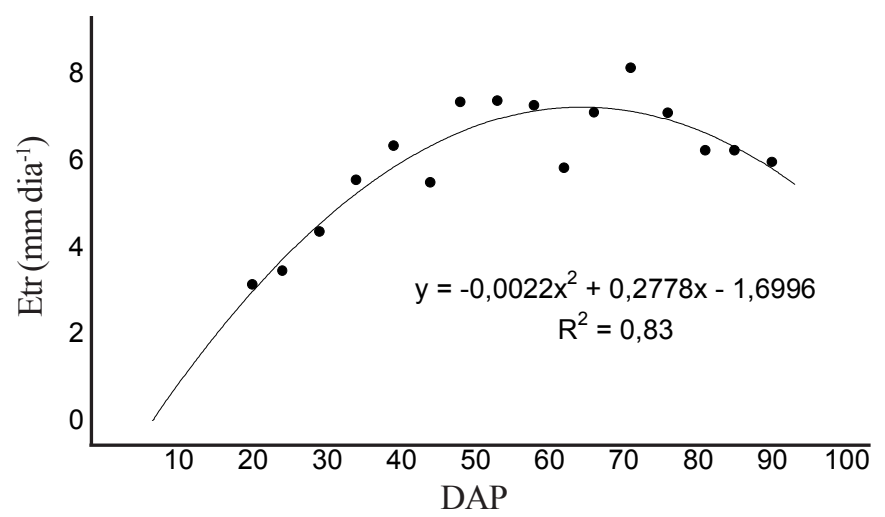

Figura 1. Evapotranspiração real do Feijão Preto, variedade Diamante Negro, em relação aos dias após plantio (DAP), em Planaltina, DF

Coeficiente de cultura: Conforme metodologia já utilizada pela EMBRAPA Cerrados e com base na evapotranspiração real média obtida no lisímetro de pesagem para os intervalos de irrigação e nas leituras do tanque classe A, para os mesmos intervalos, calcularam-se os coeficientes de cultura para cada intervalo de irrigação (Tabela 2). Para determinação dos coeficientes, utilizou-se a seguinte equação:

$$
\mathrm{kc}=\frac{E t_{\mathrm{r}}}{(\mathrm{Ekp})}
$$

onde:

kc - coeficiente da cultura

$\mathrm{Et}_{\mathrm{r}}$ - evapotranspiração real da cultura do feijão obtida no lisímetro $\left(\mathrm{mm}\right.$ intervalo $\left.{ }^{-1}\right)$ 
Tabela 2. Coeficientes de cultura para cada intervalo de irrigação: determinados na pesquisa $\left(\mathrm{kc}_{\mathrm{D}}\right)$, provenientes da FAO (Doorenbos \& Pruitt, 1977) $\left(\mathrm{kc}_{\mathrm{F}}\right)$ e propostos pela EMBRAPA/ CNPAF (Steinmetz, 1984) $\left(\mathrm{kc}_{\mathrm{E}}\right)$

\begin{tabular}{cccc}
\hline \multirow{2}{*}{ Irrigações } & \multicolumn{3}{c}{ Coeficientes de Cultura } \\
\cline { 2 - 4 } & $\mathrm{Kc}_{\mathrm{D}}$ & $\mathrm{kc}_{\mathrm{F}}$ & $\mathrm{kc}_{\mathrm{E}}$ \\
\hline $1^{\mathrm{o}}$ & 0,71 & 0,70 & 0,69 \\
$2^{\mathrm{o}}$ & 0,84 & 0,70 & 0,69 \\
$3^{\mathrm{o}}$ & 1,22 & 0,70 & 0,69 \\
$4^{\mathrm{o}}$ & 1,51 & 0,70 & 0,69 \\
$5^{\circ}$ & 1,60 & 1,15 & 0,69 \\
$6^{\mathrm{o}}$ & 1,37 & 1,15 & 0,69 \\
$7^{\mathrm{o}}$ & 1,58 & 1,15 & 1,28 \\
$8^{\circ}$ & 1,49 & 1,15 & 1,28 \\
$9^{\circ}$ & 1,56 & 1,15 & 1,28 \\
$10^{\circ}$ & 1,31 & 1,15 & 1,28 \\
$11^{\circ}$ & 1,22 & 1,15 & 1,04 \\
$12^{\circ}$ & 1,68 & 1,15 & 1,04 \\
$13^{\circ}$ & 1,43 & 1,15 & 1,04 \\
$14^{\circ}$ & 1,24 & 0,25 & 1,04 \\
$15^{\circ}$ & 1,07 & 0,25 & 1,04 \\
$16^{\circ}$ & 1,00 & 0,25 & 1,04 \\
\hline
\end{tabular}

E - evaporação do tanque classe "A" (mm intervalo $\left.{ }^{-1}\right)$

$\mathrm{Kp}$ - coeficiente do tanque Classe A adequado ao período e às condições locais do Tanque, neste caso, igual a 0,75, conforme metodologia de Doorenbos e Pruitt (1977)

\section{Descrição do modelo}

A evapotranspiração, segundo Chistiansen-Hargreaves (1969) foi calculada com base na seguinte equação:

$$
\text { Eto }=0,755 \mathrm{E}_{\mathrm{v}} \mathrm{C}_{\mathrm{T} 2} \mathrm{C}_{\mathrm{W} 2} \mathrm{C}_{\mathrm{H} 2} \mathrm{C}_{2}
$$

em que $\mathrm{E}_{\mathrm{v}}$ é a evaporação do tanque classe $\mathrm{A}$, em mm dia ${ }^{-1}$. Os demais coeficientes são adimensionais.

$$
\mathrm{C}_{\mathrm{T} 2}=0,862+0,179\left(\frac{\mathrm{T}_{\mathrm{c}}}{\mathrm{T}_{\mathrm{co}}}\right)-0,041\left(\frac{\mathrm{T}_{\mathrm{c}}}{\mathrm{T}_{\mathrm{co}}}\right)^{2}
$$

sendo $\mathrm{C}_{\mathrm{T} 2}$ um parâmetro que representa a contribuição da temperatura na evapotranspiração, donde $\mathrm{T}_{\mathrm{c}}$ é a média da temperatura $\mathrm{em}^{\circ} \mathrm{C} \mathrm{e} \mathrm{\textrm {T } _ { \text { co } }}=20^{\circ} \mathrm{C}$

$$
\mathrm{C}_{\mathrm{W} 2}=1,189-0,240\left(\frac{\mathrm{W}}{\mathrm{W}_{\mathrm{o}}}\right)+0,051\left(\frac{\mathrm{W}}{\mathrm{W}_{\mathrm{o}}}\right)^{2}
$$

com $\mathrm{C}_{\mathrm{W} 2}$ representando a contribuição da velocidade do vento na evapotranspiração, em que $\mathrm{W}$ é a velocidade do vento à $2 \mathrm{~m}$ acima do nível do solo em $\mathrm{km} \mathrm{h}^{-1} \mathrm{e}_{\mathrm{o}}=6,7 \mathrm{~km} \mathrm{~h}^{-1}$.

$$
\mathrm{C}_{\mathrm{H} 2}=0,499+0,620\left(\frac{\mathrm{H}_{\mathrm{m}}}{\mathrm{H}_{\mathrm{mo}}}\right)-0,119\left(\frac{\mathrm{H}_{\mathrm{m}}}{\mathrm{H}_{\mathrm{mo}}}\right)^{2}
$$

donde $\mathrm{C}_{\mathrm{H} 2}$ representa a contribuição da umidade relativa do ar na evapotranspiração, sendo $\mathrm{H}_{\mathrm{m}}$ é a umidade relativa média, expressada em decimal, e $\mathrm{H}_{\mathrm{mo}}=0,60$

$$
\mathrm{C}_{\mathrm{S} 2}=0,904+0,0080\left(\frac{\mathrm{S}}{\mathrm{S}_{\mathrm{o}}}\right)+0,088\left(\frac{\mathrm{S}}{\mathrm{S}_{\mathrm{o}}}\right)^{2}
$$

em que $\mathrm{C}_{\mathrm{S} 2}$ representa a contribuição dos parâmetros energéticos na evapotranspiração, donde $\mathrm{S}$ é a porcentagem de luz solar possível $(\mathrm{n} / \mathrm{N})$ expressa em decimal, e $\mathrm{S}_{\mathrm{o}}=0,80$.

\section{Avaliação do desempenho do modelo}

A comparação entre a evapotranspiração observada e a estimada envolveu dois procedimentos, sendo o primeiro uma regressão linear quadrática dos resultados, analisando-se o comportamento da curva estimada em relação à curva observada, principalmente no que diz respeito ao momento de máxima aplicação de água do modelo, o que foi obtido a partir da derivada primeira da equação resultante e o segundo uma regressão linear simples analisando-se o erro médio da estimativa, a correlação entre as evapotranspirações e o teste F, proposto por Graybill (1976). Pela aplicação do teste F ao nível de $99 \%$ de probabilidade, analisou-se a hipótese de que a evapotranspiração estimada era similar à observada, perfazendo uma linha reta, passando pela origem e de declividade igual a 45 graus, ou seja, $\beta_{0}=0$ e $\beta_{1}=1$.

\section{Ajuste do modelo}

Após a comparação entre a evapotranspiração observada e a estimada, tornou-se necessário, com base em diversos testes nos parâmetros da equação, um discreto ajuste no parâmetro energético $\left(\mathrm{C}_{\mathrm{S} 2}\right)$ do modelo Christiansen-Hargreaves, com objetivo de corrigir uma considerável subestimativa, atitude esta conveniente para a otimização do desempenho do modelo.

\section{RESULTADOS E DISCUSSÃO}

As plantas dependem da interação entre sua constituição genética e as condições ambientais, o que corresponderá a aumento ou queda de produção a depender dos défices ocorridos no período. Para a cultura em questão todo o manejo foi realizado de forma a obter o potencial produtivo da variedade, sendo assim as Figuras 1 e 2 apresentam, respectivamente, a curva ajustada da evapotranspiração real do Feijão Preto medida a partir de um lisímetro de pesagem e a curva ajustada dos coeficientes de cultura determinados na pesquisa $\left(k c_{D}\right)$, as quais serviram de referência para avaliação do desempenho do modelo.

Objetivando-se ainda avaliar o desempenho do modelo de estimativa, estabeleceram-se duas metodologias, a primeira, testando o modelo com os coeficientes de cultura (kc) determinados na pesquisa e a segunda, testando-o com coeficientes de cultura presentes na literatura [FAO $24 \mathrm{e}$ EMBRAPA/CNPAF - (Centro Nacional de Pesquisa do Arroz e Feijão) - (Tabela 2)] visando avaliar a compatibilidade desses coeficientes com a região, a época e a cultura pesquisada. 


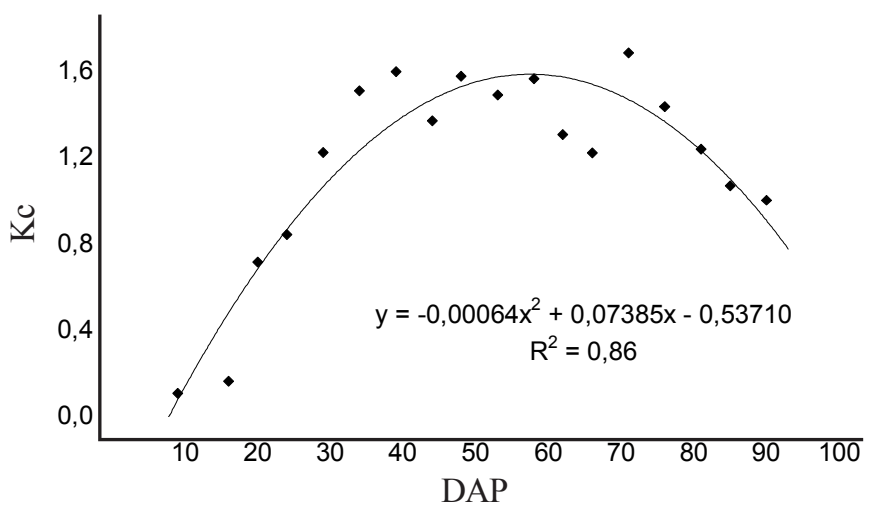

Figura 2. Coeficiente de cultura para o Feijão Preto, variedade Diamante Negro, em relação aos dias após o plantio (DAP) em Planaltina, DF

Muito embora os coeficientes de cultura para o feijão (variedade Diamante Negro) pudessem ser obtidos a partir da equação resultante da regressão linear dos dados (Figura 2), optou-se em testar o modelo com coeficientes de cultura determinados conforme a metodologia descrita, com o propósito de testar o modelo sem os benefícios do ajuste dos coeficientes e com a metodologia normalmente utilizada na Embrapa Cerrados.

Pela Figura 3 verifica-se um limitado desempenho do modelo Chistiansen-Hargreaves $(\mathrm{CH})$, pois percebe-se que, apesar da ótima precisão, $\mathrm{R}^{2}=0,95$, a exatidão foi baixa, $\beta_{0}=0,3328 \mathrm{e}$ $\beta_{1}=0,8272$, mostrando considerável tendência em subestimar $\mathrm{aEt}$.

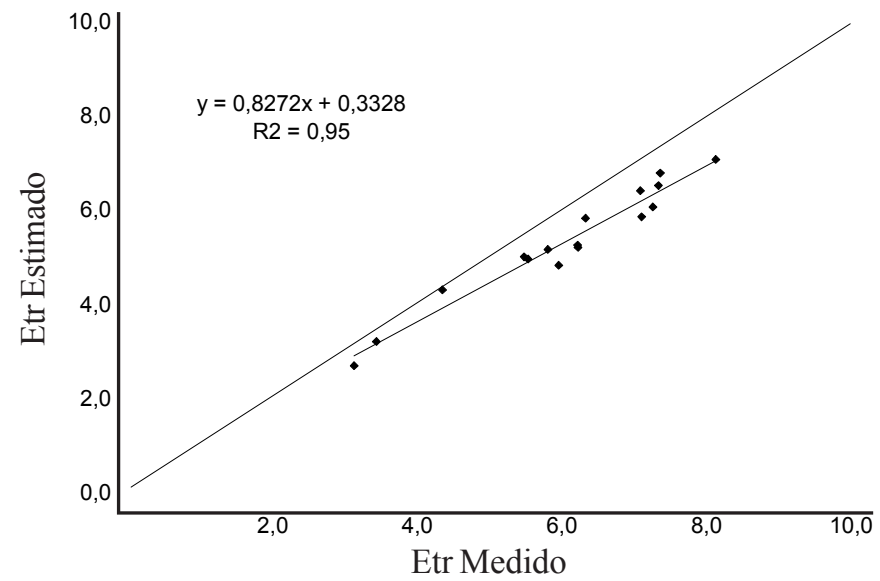

Figura 3. Relação entre a $\mathrm{Et}_{\mathrm{r}}$ estimada a partir do modelo Christiansen-Hagreaves, e medida em lisímetro de pesagem, em Planaltina, DF, para o Feijão Preto

Nas Tabelas 3 e 4 nota-se, respectivamente, um erro médio da estimativa de $-11,78 \%$, com F significativo (diferem significativamente ao nível de $99 \%$ de probabilidade) o que reforça o mau desempenho do modelo; entretanto, observando a Figura 4, nota-se que o ponto de máxima evapotranspiração, ocorrido aos 63 DAP, foi exatamente igual ao observado nos dados do lisímetro, demonstrando perfeita conformação da curva estimada em relação à observada. Esta observação, associada ao período analisado (seco) e aos testes nos parâmetros do modelo, indicou a necessidade de aumentar a contribuição do
Tabela 3. Erros médios na estimativa da $\mathrm{Et}_{\mathrm{r}}$ do feijoeiro obtidos com a aplicação dos seis modelos em Planaltina, DF

\begin{tabular}{lccc}
\multirow{2}{*}{ Modelo } & \multicolumn{2}{c}{$\begin{array}{c}\text { Evapotranspiração } \\
\text { Real* }(\mathrm{mm})\end{array}$} & $\begin{array}{c}\text { Erro Médio } \\
\text { da Estimativa (\%) }\end{array}$ \\
\cline { 2 - 3 } & Medida & Estimada & \\
\hline $\begin{array}{c}\text { Chistiansen- } \\
\text { Hargreaves }\end{array}$ & 96,70 & 85,31 & $-11,78$ \\
$\begin{array}{c}\text { Chistiansen- } \\
\text { Hargreaves (ajustado) }\end{array}$ & 96,70 & 98,11 & 1,46 \\
\hline
\end{tabular}

* Et resultante do somatório das médias diárias observadas por intervalo de irrigação

Tabela 4. Valores da análise de regressão simples entre a $\mathrm{Et}_{\mathrm{r}}$ estimada pelos seis modelos e a Et do Feijão Preto medida em lisímetro de pesagens em Planaltina, DF

\begin{tabular}{lcccc} 
& \multicolumn{3}{c}{ Coeficientes } & F de \\
\cline { 2 - 4 } \multicolumn{1}{c}{ Modelos } & $\beta_{0}$ & $\beta_{1}$ & $\mathrm{R}^{2}$ & Graybill \\
\hline $\begin{array}{c}\text { Chistiansen- } \\
\text { Hargreaves }\end{array}$ & 0,3328 & 0,8272 & 0,95 & $73,14^{*}$ \\
$\begin{array}{c}\text { Chistiansen- } \\
\text { Hargreaves (ajustado) }\end{array}$ & 0,3828 & 0,9513 & 0,95 & 0,77 \\
\hline
\end{tabular}

* Significativo ao nível de $99 \%$ de probabilidade

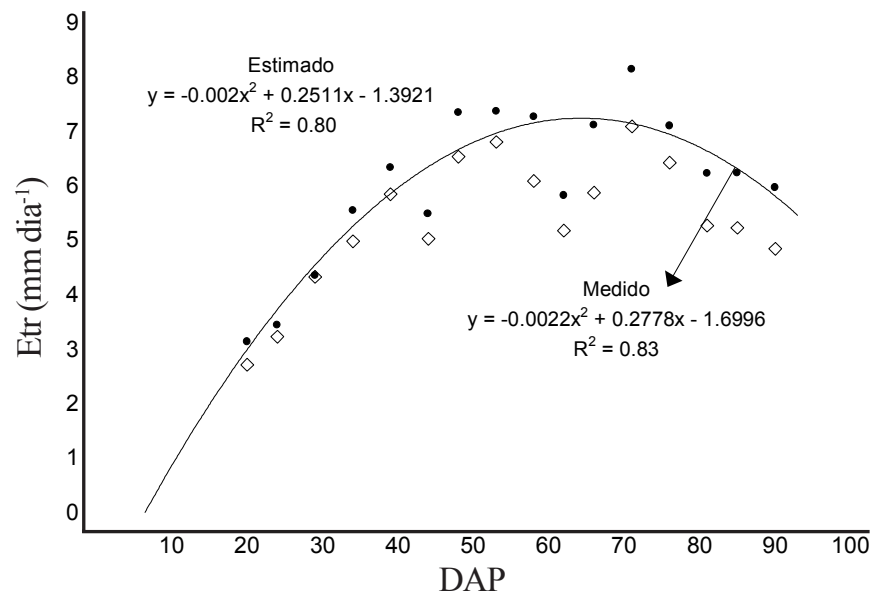

Figura 4. Comparação entre a $\mathrm{Et}_{\mathrm{r}}$ estimada a partir do modelo Chistiansen-Hargreaves, e medida em lisímetro de pesagem, em relação aos dias após plantio (DAP), em Planaltina, DF, para o Feijão Preto

termo energético no modelo o que foi possível com a redução no valor do parâmetro $S_{0}$ de 0,8 para 0,5 . Este ajuste proporcionou uma melhoria significativa no desempenho, como pode ser observado na Figura 5, onde a correlação se manteve alta, $\mathrm{R}^{2}=0,95$, e a concordância aumentou consideravelmente $\left(\beta_{0}=\right.$ 0,3828 e $\left.\beta_{1}=0,9513\right)$. Este fato proporcionou uma grande redução no erro médio da estimativa de $-11,78 \%$ para $1,46 \%$ (Tabela 3 ) e um F de Graybill não significativo (Tabela 4), o que permite confirmar o ótimo desempenho do modelo $\mathrm{CH}$, desde que a contribuição do termo energético seja incrementada.

Pela Figura 6, que apresenta a comparação entre os dados médios de $\mathrm{Et}_{\mathrm{r}}$ medidos no lisímetro e estimados pelo modelo Chistiansen-Hargreaves ajustado $(\mathrm{CH})$ testado com os coeficientes de cultura determinados na pesquisa (CHkcD), obtidos segundo a metodologia do FAO24 (CHkcF) e fornecidos pela Embrapa Arroz e Feijão (CHkcE) em relação a dias após plantio, verifica-se que o $\mathrm{kc}_{\mathrm{F}}$ desfavoreceu o 


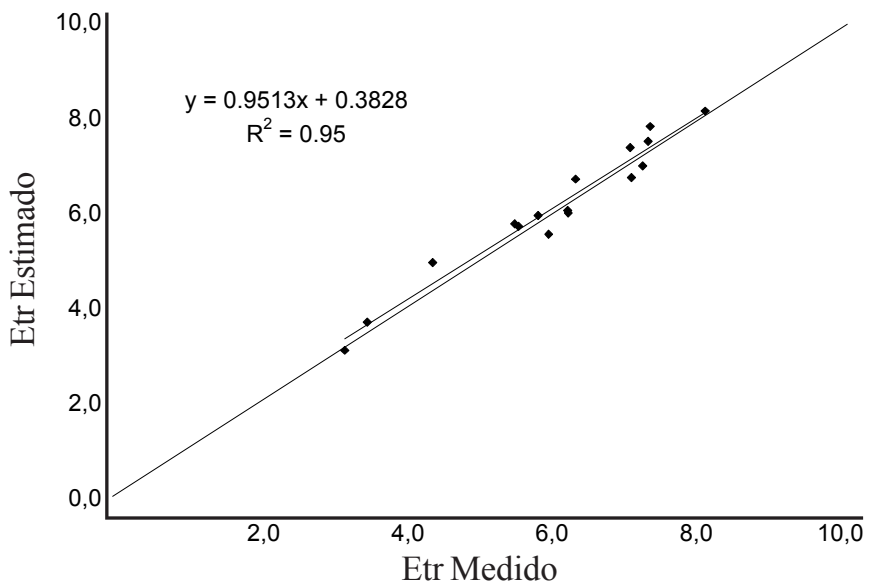

Figura 5. Relação entre a $\mathrm{Et}_{\mathrm{r}}$ estimada a partir do modelo Christiansen-Hagreaves ajustado, e medida em lisímetro de pesagem, em Planaltina, DF, para o Feijão Preto

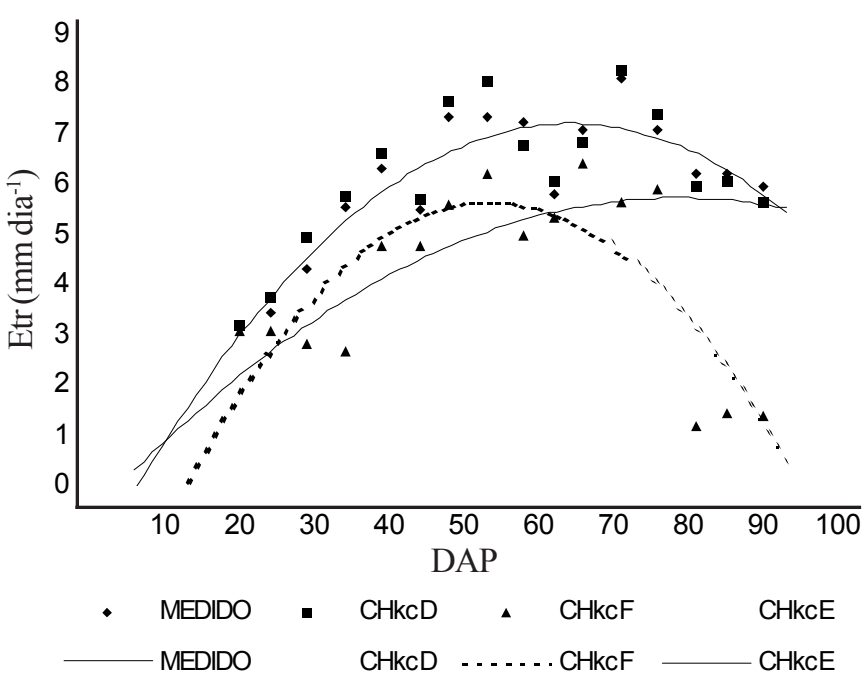

Figura 6. Comparação entre a $\mathrm{Et}_{\mathrm{r}}$ estimada a partir do modelo Christiansen-Hargreaves ajustado calculado com diferentes coeficientes de cultura, e medida em lisímetro de pesagem, em relação aos dias após plantio (DAP), em Planaltina, DF, para o Feijão Preto

desempenho do modelo $\mathrm{CH}$, já que estimou um ponto de máxima evapotranspiração no momento da floração, aos 54 DAP, ao contrário do que foi observado no lisímetro, em que a maior demanda hídrica do feijoeiro ocorreu aos 63 DAP, exatamente no início do enchimento das vagens. Este resultado, associado aos dados subestimados proporcionados pelo $\mathrm{kc}_{\mathrm{F}}$, resultaria em aplicações de lâminas inferiores às requeridas pela cultura o que proporcionaria redução significativa na produtividade; assim, pode-se inferir que, para o período seco da região do Cerrado, é inviável a utilização do modelo Chistiansen Hargreaves ajustado com os coeficientes sugeridos pela FAO; da mesma forma, percebe-se que o $\mathrm{kc}_{\mathrm{E}}$ limitou o desempenho do modelo $\mathrm{CH}$, já que apresentou dados subestimados durante todo o ciclo da cultura e ponto de máxima evapotranspiração aos 78 DAP, aproximadamente no início da maturação fisiológica dos frutos, fato que comprometeria o desenvolvimento da cultura, prejudicando a produtividade e a qualidade dos grãos.

Deve-se levar em conta que o mau desempenho proporcionado pelos coeficientes propostos por Steinmetz (1984)
(Embrapa) e obtidos a partir de Doorenbos \& Pruitt (1977) (FAO) comprova que os coeficientes de uma cultura não podem ser generalizados para diversas regiões e períodos, o que possibilita afirmar que existe uma grande possibilidade de que os coeficientes de cultura encontrados nesta pesquisa, possam também limitar o desempenho do modelo nos demais períodos do ano na região do Cerrado.

\section{CONCLUSÕES}

1. O modelo de Chistiansen-Hargreaves (1969), na sua forma original, subestimou significativamente a evapotranspiração real do Feijão Preto; todavia, seu desempenho foi otimizado com o aumento da contribuição do termo energético no modelo.

2. O bom desempenho do modelo Chistiasen-Hargreaves ajustado, qualifica-o como uma alternativa viável para o manejo de irrigação do Feijão Preto no período seco da região do Cerrado, mas, sua utilização prática encontra-se associada aos coeficientes de cultura determinados nesta pesquisa, os quais podem ser estimados a partir da equação apresentada na Figura 2.

3. Os coeficientes de cultura obtidos segundo FAO-24 e propostos por Steinmetz (1984) são inadequados para as condições analisadas, pois limitaram o desempenho do modelo, subestimando significativamente $\mathrm{Et}_{\mathrm{r}}$ do feijoeiro.

\section{LITERATURA CITADA}

Brasil. Ministério da Agricultura. Departamento de transferência e comercialização de tecnologias. Recomendações técnicas para o cultivo do feijão. Brasília, EMBRAPA-SPI, 1996. 32p.

Chistiansen, J.E.; Hargreaves, G.H. Irrigation requirements from evaporation. Transactions of International Commission on Irrigation and Drainage, New York, v.3, n.23.p.569-596. 1969.

Doorenbos, J.; Pruitt, W.O. Crop water requirements. Irrigation and Drainage, Paper 24. Rome: Food and Agriculture Organization of the United Nations, 1977. 144p.

Figuerêdo, S.F.; Guerra, A.F.; Silva, D.B. da; Antonini, J.C. dos; Rodrigues, G.C. Manejo de irrigação e fertilização nitrogenada na cultura do feijão. In: EMBRAPA. Centro de Pesquisa Agropecuária dos Cerrados (Planaltina, DF). Planaltina, 1997. p. 95-97. Relatório Técnico Anual 1991 a 1995

Graybill, F.A. Theory and application of the linear model. s. 1. Belmont Duxdury, 1976. 704p.

Guerra, A.F.; Silva, D.B. Manejo de irrigação e fertilização nitrogenada para cevada de seis fileiras na região do cerrado. In: Reunião Anual de Pesquisa de Cevada, 18, 1998, Passo Fundo. Anais... Passo fundo, EMBRAPA, 1998, p.365-371.

Jensen, M.E.; Burman, R.D.; Allen, R.G. Evapotranspiration and irrigation water requirements. New York, ASCE. 1989. p. 332, Manuals and reports 70

Macedo, J. Apresentação. In: Simpósio sobre Cerrado, 7, 1995, Brasília. Anais... Planaltina, Embrapa-CPAC, 1997, p.13.

Roston, A.J. Feijão. CATI. Secretaria de Agricultura e Abastecimento do Governo do Estado de São Paulo, Campinas. Boletim Técnico 199, 1990, 18p.

Steinmetz, S. Evapotranspiração máxima no cultivo do feijão de inverno. Goiânia, EMBRAPA-CNPAF, 1984. p.11. EMBRAPA - CNPAF Pesquisa em Andamento, 47p. 